\title{
Implementation of red black strategy to quarter sweep iteration for solving first order hyperbolic equations
}

\begin{abstract}
Our previous researches have shown the extraordinary performance of quarter sweep iteration to speed-up the original method by four times. In this paper, an experimental study is conducted to show the efficiency of the red-black quarter-sweep iteration by using the CrankNicolson $(\mathrm{CN})$ finite difference approximation scheme to obtain numerical solutions of the first order hyperbolic equation. In this paper, the implementation of the red-black strategy to full-sweep Gauss-Seidel (FGS-RB), half-sweep Gauss-Seidel (HGS-RB), and the quartersweep Gauss-Seidel (QGS-RB) methods will be discussed. Finally, through numerical results obtained, the QGS-RB iterative method has been shown to be the most superior method compared to FGS-RB and HGS-RB methods.
\end{abstract}

